\section{Eigenregions for Image Classification}

\author{
Clément Fredembach, Student Member, IEEE, \\ Michael Schröder, Member, IEEE, and \\ Sabine Süsstrunk, Member, IEEE
}

\begin{abstract}
For certain databases and classification tasks, analyzing images based on region features instead of image features results in more accurate classifications. We introduce eigenregions, which are geometrical features that encompass area, location, and shape properties of an image region, even if the region is spatially incoherent. Eigenregions are calculated using principal component analysis (PCA). On a database of 77,000 different regions obtained through the segmentation of 13,500 real-scene photographic images taken by nonprofessionals, eigenregions improved the detection of localized image classes by a noticeable amount. Additionally, eigenregions allow us to prove that the largest variance in natural image region geometry is due to its area and not to shape or position.
\end{abstract}

Index Terms-Eigenregions, image classification, region analysis, image features.

\section{INTRODUCTION}

MANY imaging applications, such as automatic color correction (ACC) or content-based image retrieval (CBIR), are based on successful image classification [2], [4], [14], [18]. It is evident that the performance of these algorithms is directly linked to the performance of the classification. Thus, in order to be effective, image classification algorithms need features that well express relevant image properties. These features are often calculated based on the whole image. However, dependent on the database and task, a region-based approach that uses more localized information can improve image classification results [5], [6], [7], [13].

In this paper, we introduce eigenregions which are geometrical features that encompass area, location, and shape properties of a region. Eigenregions are obtained by analyzing segmented image regions with Principal Component Analysis (PCA). Principal component analysis has already successfully been implemented in image classification for many tasks (see [15], [19] for examples), but usually on the whole image. As opposed to other geometrical region features [8], [10], [17], eigenregions can be used and result in significant classification improvement even if the image regions are spatially incoherent. They are also visually significant and computationally efficient. Another key result obtained with eigenregions is that for a large data set of natural images, the largest variance in region geometry is due to the area and not to shape or position. We tested the performance of eigenregions in an image classification experiment, where the goal was to correctly identify semantic image classes, such as "blue sky," "skin tone," and "vegetation." The data set consisted of 13,500 real-scene photographic images taken by nonprofessionals. After segmenting the image into regions, we calculate region features, classify the regions, calculate the corresponding image features and, finally, classify the images (see Fig. 1). We found that the addition of eigenregions to the feature vector improves region classification results for localized classes, i.e., for region classes usually localized in a specific part of an image, such as

- C. Fredembach is with the School of Computing Sciences, University of East Anglia, Norwich, NR4 9TJ UK.

E-mail: clement.fredembach@a3.epfl.ch.

- M. Schröder is with the Swiss Technology Consulting Group AG, Zurich, Switzerland.

- S. Süsstrunk is with the Audiovisual Communications Laboratory (LCAV), Swiss Federal Institute of Technology (EPFL), Lausanne, Switzerland. E-mail: michael.schroeder@alumni.ethz.ch.

Manuscript received 24 Sept. 2003; revised 1 Mar. 2004; accepted 21 Apr. 2004. Recommended for acceptance by $V$. Govindaraju.

For information on obtaining reprints of this article, please send e-mail to: tpami@computer.org, and reference IEEECS Log Number TPAMI-0285-0903. sky regions. We also show the relevance of eigenregions by classifying regions using only eigenregions as features, where they achieved a very respectable success rate. Finally, the image classification rate based on region features is significantly better than the classification based on image features only.

The paper is organized as follows: Section 2 discusses the image segmentation algorithms used. Section 3 discusses the drawback of existing geometrical features, explains how eigenregions are obtained, and demonstrates their significance. Section 4 illustrates the usefulness of eigenregions for region classification, Section 5 explains how to obtain image classification from region classification, and Section 6 concludes the paper.

\section{ImAge Segmentation}

To be able to extract region features, images need first to be segmented into meaningful regions. We chose the Dominant Colors in Lab (DC Lab) method, based on the MPEG-7 guidelines [12]. It uses a k-means powered algorithm to segment images according to (CIE) Lab clusters [21]. The $k$ parameter, representing the maximal number of possible clusters, is fixed to 8. Arguments for using this algorithm over other available ones [1], [11] include its computational speed (real time processing) and its ease of use, while still providing good quality segmentation. However, DC Lab regions are not spatially coherent (see Fig. 2) which prevents the use of many existing geometrical features (see Section 3).

For comparison purposes, we also calculated eigenregions with image regions obtained via edgeflow segmentation [11]. Since edgeflow regions are visually different from DC Lab, we compared eigenregions to find if the geometrical characteristics of the regions are due to the segmentation algorithm or not. We found that the eigenregions are similar in both cases, suggesting that these structural features are independent of the segmentation. The results presented in the rest of the paper are based on DC Lab regions.

\section{EIGENREGIONS}

One of the major requirements for a robust classification is to have a set of relevant and reliable features that will conveniently describe a region. Color and texture features have received far more attention and usually perform better than geometrical features. It was found that the latter can either be too general or too precise [10]. Some geometrical properties, such as region position, does not in general fulfill continuous or Gaussian assumptions required by some classification algorithms [17]. Moment analysis could be used, but it is difficult to determine a priori how many are necessary for a good classification [8], [10]. Another drawback is that if the image segmentation algorithm results in regions that are not spatially coherent, the perimeter or radii of a region cannot be used as parameters [3]. Other geometrical properties could be utilized in this case, such as the compactness or the positions of a region in the image. These features are, however, too limited to improve an already high classification rate on our image database [5], whereas the proposed eigenregions do significantly improve the image classification (see Section 4).

Eigenregions are obtained by calculating the principal components of the region locations. The segmented images have a size of $64 \times 48(3,072)$ pixels, resulting in a dimensionality too large to undertake a PCA. We therefore started by downsampling the regions to a $5 \times 5$ area, such as shown in Fig. 3 . Since the area of interest is the coverage of a pixel by a region, the downsampling procedure determines which percentage of a new "pixel" was originally covered by the region. Let $R$ be a region, $I$ the image to which the region belongs, and $\rho$ be a pixel. Consider the downsampling procedure:

$$
\begin{array}{r}
\forall \rho \in I: I(\rho)=1 \text { if } \rho \in R, 0 \text { otherwise } \\
I_{\text {down }}(i)=\frac{1}{\beta} \sum_{j=1}^{\beta} I(\beta(i-1)+j),
\end{array}
$$




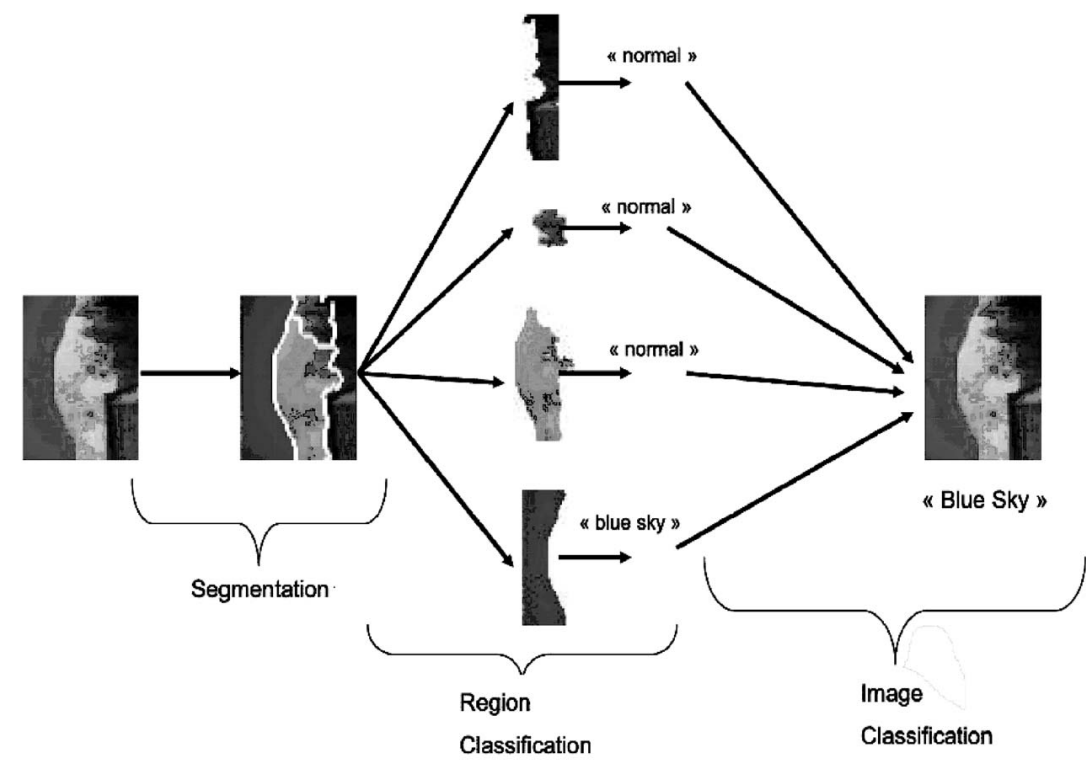

Fig. 1. This figure illustrates the major steps of the classification framework. First, the image is segmented into several regions. Using region-specific features, the regions are classified in several semantic classes. The results from region classification are then used to obtain a robust image classification.

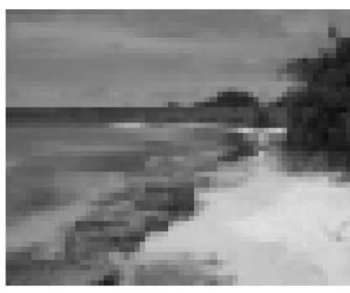

(a)

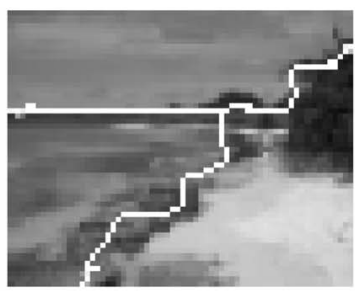

(b)

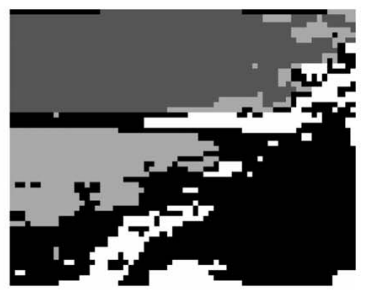

(c)

Fig. 2. (b) An image segmented with edgeflow and (c) DC Lab. Contrarily to Edgeflow, DC Lab regions, represented by different gray levels, are not spatially coherent.

where $\beta=\left\lfloor\frac{3,072}{25}\right\rfloor$ is the downsampling factor and $I_{\text {down }}$ is the downsampled image that will be used in the principal component analysis. Note that we investigated a number of downsampling factors, ranging from $3 \times 3$ to the entire image. Downsampling of $5 \times 5$ and higher lead to the same visual and mathematical results. We present the results for a $5 \times 5$ downsampling, which is the most computationally efficient implementation.

The eigenregions are then calculated on a set of 77,776 $(N)$ regions obtained via the segmentation of 13,500 real-scene photographic images taken by nonprofessionals, i.e., consumer images.

The general PCA analysis is as follows: let $X$ be the data matrix ( $N$ vectors of length 25$)$ and let $\bar{X}(1 \times 25)$ be the mean over all observations of $X . Y$ is defined as $X$ with $\bar{X}$ subtracted from each of its columns. The covariance matrix is $C=Y \cdot Y^{T}$, and the single value decomposition of $C$ yields $C=V \cdot \Lambda \cdot V^{T}$, where $V$ is the eigenvector matrix and $\Lambda$ is the diagonal matrix containing the eigenvalues.

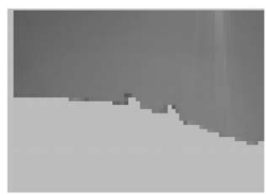

(a)

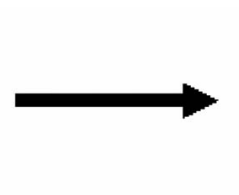

西

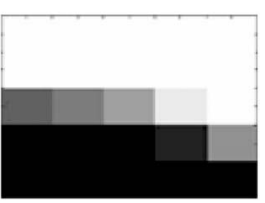

(b)
Fig. 3. (a) Downsampling of a region to a $5 \times 5$ image. The gray values of the down-sampled image display proportional coverage, from 100 percent (white) to 0 percent (black).
Using this method, the first eigenfeature (the one corresponding to the largest eigenvalue) will be in the direction of the largest variance. Of importance to the classification is the fact that the eigenvectors are orthogonal. This implies that they can all be used as features in the selection process since they are independent.

Fig. 4 shows the 25 eigenregions obtained using this method. The reconstruction rates can be observed in Fig. 5. From a visual point of view, a semantical meaning can be given to the first five eigenregions. The centered vertical and horizontal split (eigenregions 2 and 3) are due for the most part to landscape photographs having a horizon line. Eigenregions 4 and 5 represent the distinction between an object and a background. Since those distinctions strongly depend on the nature of the photographed object (shape, color, sharpness, etc.), it seems normal that those regions come after the landscape-type ones, as the latter are much more common in real-scene photography. The remaining eigenregions cannot easily be given semantic meaning. One explanation is that DC Lab also outputs a lot of small sized regions that can appear noisy when displayed as such.

Interestingly, the first eigenregion appears to be a "mean." Recall that the mean of all regions was removed prior to the principal component calculation. Thus, the largest variation of the regions is in the direction of the mean, even though it is removed. By letting $C=X \cdot X^{T}$ instead of $Y \cdot Y^{T}$, we can apply a singular value decomposition instead of a standard principal component analysis to the data set. The results of this method are illustrated in Fig. 6.

Comparing Figs. 4 and 6, we can observe that removing the mean from the data does not alter the obtained eigenregions. Given that the union of an image's regions is the image, we can compute the average region size by dividing the total number of images by 


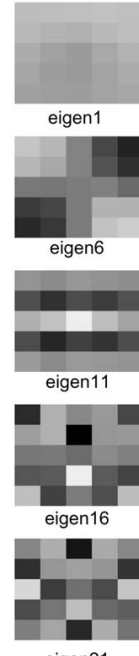

eigen21
Eigenregions with mean removed from data

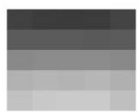

eigen2

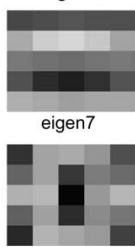

eigen12

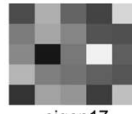

eigen17

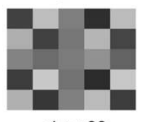

eigen22

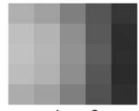

eigen3

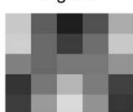

eigen8

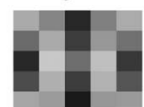

eigen13

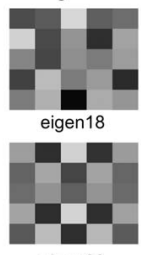

eigen23

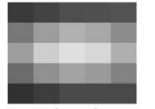

eigen4

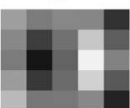

eigen9

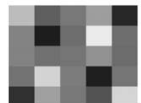

eigen14
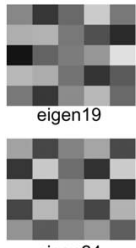

eigen24

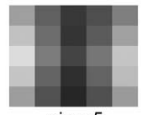

eigen5

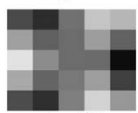

eigen10

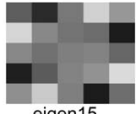

eigen15
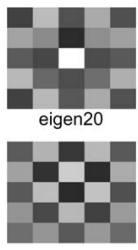

eigen 25

Fig. 4. DC Lab eigenregions. Coverage values range from -1 (black) to +1 (white) Those regions express the most common structure of segmented images. Relevant semantic regions include top/bottom (eigen 2), left/right (eigen 3), and various center/surround interactions (eigen 4 and 5 ).

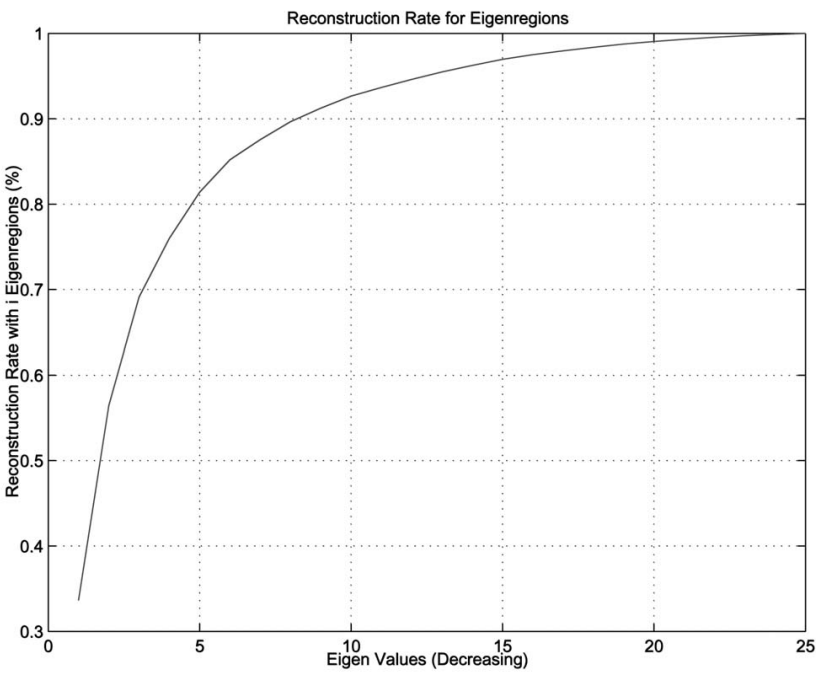

Fig. 5. Eigenregion reconstruction rates.

the total number of regions. In our case, we have 77,776 regions from 13,512 images, yielding a value of 0.17 . This value is not small enough to justify the fact that removing it bears no influence on the final result.

We can prove that for our experimental set-up, the greatest variance of a region's structure is in the same direction as the mean. Let $X, \bar{X}, Y$, and $N$ be as previously defined. From the singular value decomposition, we have:

$$
\begin{aligned}
& X \cdot X^{\mathrm{T}}=U \cdot D \cdot U^{\mathrm{T}}=\sum d_{i i} \cdot u_{i} \cdot u_{i}^{\mathrm{T}}, \\
& Y \cdot Y^{\mathrm{T}}=V \cdot \Lambda \cdot V^{\mathrm{T}}=\sum \lambda_{i i} \cdot v_{i} \cdot v_{i}^{\mathrm{T}} .
\end{aligned}
$$

Expanding (2) yields:

$$
\begin{aligned}
Y \cdot Y^{\mathrm{T}]} & =\left(X-\bar{X} e^{\mathrm{T}}\right) \cdot\left(X-\bar{X} e^{\mathrm{T}}\right)^{\mathrm{T}} \\
& =X X^{\mathrm{T}}-X e \bar{X}^{\mathrm{T}}-X^{\mathrm{T}} e^{\mathrm{T}} \bar{X}-N \bar{X} \bar{X}^{\mathrm{T}} \\
& =X \cdot X^{\mathrm{T}}-N \cdot \bar{X} \cdot \bar{X}^{\mathrm{T}},
\end{aligned}
$$

where $e$ is a vector of size $1 \times 25$ containing only 1 's. If the first eigenvector is in the same direction as the mean, then:
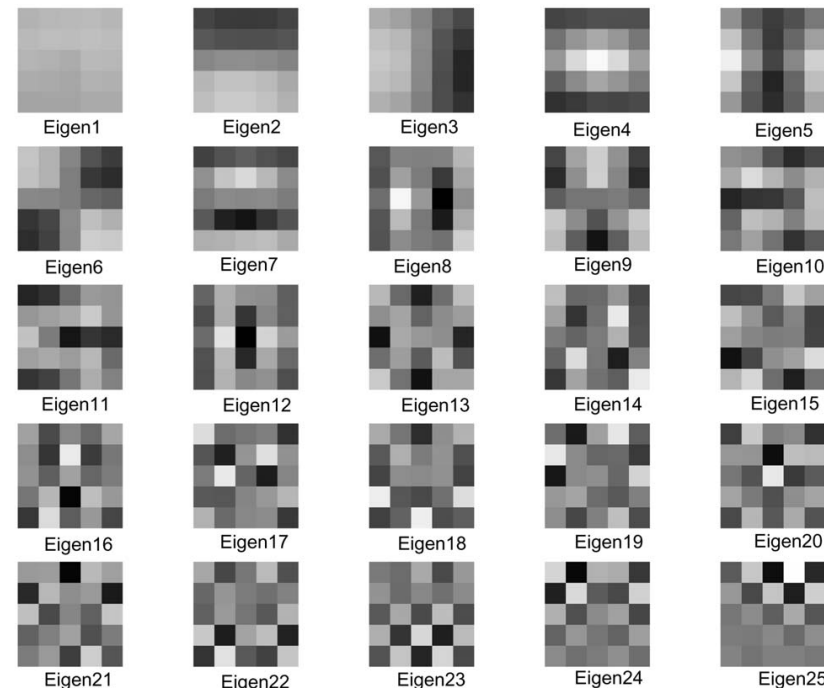

Eigen3

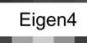

Eigen5
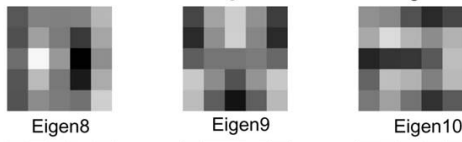

Eigen10
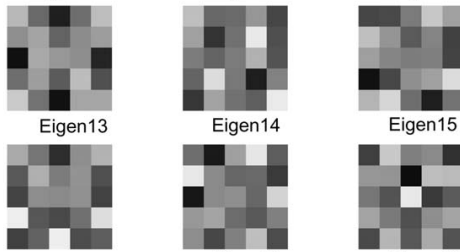

Eigen18

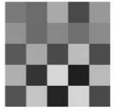

Eigen19
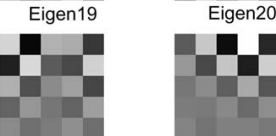

Eigen23

Eigen24

Eigen25

Fig. 6. DC Lab "eigenregions" without the mean removed from each column of $X$. The overall structure of the regions does not significantly change, indicating that the greatest variance of the data is in the direction of the mean.

TABLE 1

Eigenvalues for the Two Different Variants, without $\left(X \cdot X^{\mathrm{T}}\right)$ and with $\left(Y \cdot Y^{\mathrm{T}}\right)$ the Mean Removed

\begin{tabular}{|c|c|c|c|}
\hline$\Lambda(\times)$ & $X \cdot X^{\mathrm{T}}$ & $Y \cdot Y^{\mathrm{T}}$ & Difference \\
\hline$\lambda_{1}$ & 79,835 & 49,225 & $30^{\prime} 610$ \\
\hline$\lambda_{2}$ & 24,075 & 24,032 & 43 \\
\hline$\lambda_{3}$ & 11,108 & 11,085 & 23 \\
\hline$\lambda_{4}$ & 8,519 & 8,519 & 0 \\
\hline$\lambda_{5}$ & 6,324 & 6,310 & 14 \\
\hline$\lambda_{6}$ & 4,680 & 4,680 & 0 \\
\hline$\lambda_{7}$ & 3,588 & 3,587 & 1 \\
\hline$\lambda_{8}$ & 2,794 & 2,794 & 0 \\
\hline$\lambda_{9}$ & 2,714 & 2,709 & 5 \\
\hline
\end{tabular}

For $i>9$, the difference for $\lambda_{i}$ is zero.

$$
\bar{X} \cdot \bar{X}^{\mathrm{T}}=c \cdot u_{1} \cdot u_{1}^{\mathrm{T}},
$$

where $c$ is the norm of $\bar{X}$. If removing the mean does not change the direction of the first eigenvector, the other eigenvectors will not be altered either. The largest eigenvalue will decrease by $N \cdot c$ and the other eigenvalues will stay the same. Using (5), we have:

$$
\begin{aligned}
Y \cdot Y^{\mathrm{T}} & =X \cdot X^{\mathrm{T}}-N \cdot \bar{X} \cdot \bar{X}^{\mathrm{T}} \\
\sum \lambda_{i i} \cdot v_{i} \cdot v_{i}^{\mathrm{T}} & =\sum d_{i i} \cdot u_{i} \cdot u_{i}^{\mathrm{T}}-N \cdot c \cdot u_{1} \cdot u_{1}^{\mathrm{T}},
\end{aligned}
$$

which yields to, if the hypothesis is correct:

$$
\begin{aligned}
& \lambda_{11}=d_{11}-c \cdot N \\
& \forall i \geq 2, \lambda_{i i}=d_{i i} .
\end{aligned}
$$

Calculating the principal components in both cases enables us to compare the eigenvalues and experimentally verify that it corresponds to the theory. Table 1 regroups the eigenvalues obtained with and without the mean removed. The norm of the mean vector being $c=0.393$, it is easy to verify that $\lambda_{11}=d_{11}-c N$. The table entries also show that all the other eigenvalues are the same, within numerical approxiamtion. 


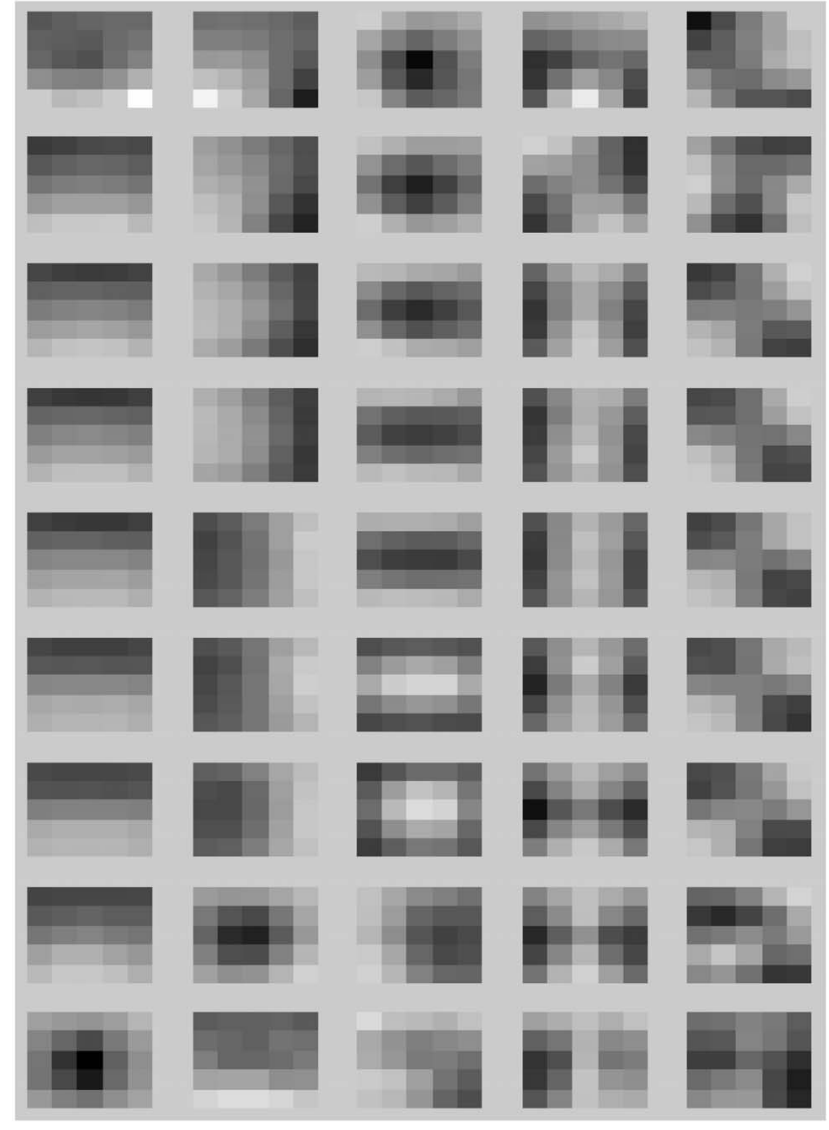

Fig. 7. First five eigenregions using PCA on nine area bins. Bins from smallest to largest area are ordered from top to bottom. The "mean-like" eigenregion does not appear anymore and the others are similar to those obtained on all regions (see Figs. 4 and 6).

\subsection{Area as the First Eigenvector}

We have shown that the greatest variance in the data is in the direction of the mean. Our hypothesis is that this variance is due to the relative area of a region. Eigenregions can be seen as features encompassing shape, location, and size properties. It is, however, unlikely that either shape or location are involved in this variance since the first eigenregion has no defined shape nor location.

We verified our assumption that the first eigenvector is an indicator of area, and not shape or location by separating the regions according to their area. We define the relative area of a region $a_{R_{i}}$ as $a_{R_{i}}=\left\|x_{i}\right\|$, where $R_{i}$ is a region of the image $I$, and $x_{i}$ the $i$ th column of $X$ that corresponds to $R_{i}$. We then create $i$ histogram bins $A_{i}$ such that:

$$
A_{i}=\left[a_{R_{j}} \mid b_{i} \leq a_{R_{j}} \leq c_{i}\right], \forall R_{j} .
$$

Using the area distribution of the regions, we obtained nine bins. Expressed in terms of the regions relative area, they are: $0-0.05$, $0.05-0.1,0.1-0.15,0.15-0.2,0.2-0.3,0.3-0.4,0.4-0.6,0.6-0.8$, and $0.8-1$. $Y_{A_{i}}$ denotes the data matrix of regions that are in the area bin $A_{i}$ with their mean removed. For each $A_{i}$, we have $C_{A_{i}}=Y_{A_{i}} \cdot Y_{A_{i}}^{T}$ and obtain $V_{A_{i}}$ and $\Lambda_{A_{i}}$ with the principal component analysis of $C_{A_{i}}$.

Fig. 7 shows the first five eigenregions for all nine area bins. The overall structure of those eigenregions is very similar to those obtained over all regions, with the exception of the first eigenregion of $X$. Consequently, removing a possible variation of the area also removes the mean-like eigenregion, thereby indicating that the largest variance of a region structure is in fact the region area. We further verified this by computing the inner product of $V_{A_{i}}$ with the first eigenvector of $X$, and found them to be almost orthogonal.
TABLE 2

Classification Success Rates (Average of Correct Positive and Correct Negative Rates) Using only Eigenregions as Features

\begin{tabular}{|c|c|c|c|}
\hline Class & Success Rate & Best Eigenregion & Best feature rate \\
\hline Skin & 0.864 & eigen4 (center) & 0.817 \\
\hline Vegetation & 0.667 & eigen2 (top/bottom) & 0.58 \\
\hline Blue sky & 0.832 & eigen2 (top/bottom) & 0.805 \\
\hline
\end{tabular}

Detection rates are very good for localized classes (skin, blue sky), but not for nonlocalized ones (vegetation). The second column (Success Rate) is obtained using all eigenregions; the fourth one (Best feature rate) is the maximum rate using a single eigenregion only.

As a result, we can conclude that the largest variance of natural image region structure is the region's area. This result is, of course, dependent on our image data set. However, this does not mean that using eigenregion features are not usable for image classification of natural images, as is shown in the next section, but that the inherent variation of natural image structures is principally due to the regions area.

\section{EigenRegions in Region Classification}

We tested the usefulness of eigenregions in an image classification task. The primary goal was to improve the performance of class specific automatic color correction (ACC) [16]. The considered classes blue sky, skin tones, and vegetation are critical for the performance of ACC. The classification algorithm used in this method is a multivariate gaussian analysis based on the maximum a posteriori rule [8], which was chosen for its robustness and scalability. This is a supervised classification algorithm, which implies that the class labels have to be defined beforehand.

A set of features is given to the classifier that then retains the relevant ones. If a feature does not actively help to differentiate two classes, it will not be selected. The "surviving" features therefore provide the best classification performance (for more details about the classification framework, see [6]).

Besides eigenregions, color and texture features were also calculated for each region. Color features include mean and standard deviation of R, G, B, L, a, and b. As texture features, we chose seven of the Haralick features [9] that are based on a GrayLevel Co-occurrence Matrix (GLCM). Energy, Contrast, Homogeneity, Inverse Different Moment, and Entropy have been defined by Haralick and two, Cluster Shade and Cluster Prominence, have been added later by Smits and Annoni [20]. In addition, we also use the mean amplitude spectrum of the Fourier Transform of a region's luminance, which represents a texture intensity measure.

We assessed the discriminative power of eigenregions by classifying regions using only eigenregions and using eigenregions in conjunction with the other acquired features. In the first case, the input to the algorithm is the approximation of the regions using the 10 first eigenregions. The classifier then determines which of those features are useful for classification, as well as the resulting false positive and false negative rates. Numerical results are listed in Table 2. We found that classification rates for skin and blue sky regions are better than for vegetation regions. This can be explained by the fact that vegetation is not a "localized" class. Vegetation can occur anywhere in the image, therefore rendering geometrical features less meaningful, whereas skin and sky regions will have a tendency to be located in a specific part of an image (top and center, respectively).

For the second experiment, we used a feature vector composed of the color, texture, and geometrical features [5], [6] to which the 10 eigenregion features were added. Table 3 shows that despite the already solid results obtained, the use of eigenregions permitted a noticeable improvement of the classification of sky and skin regions. The results show that even though the reconstruction rate 
TABLE 3

Regions Classification Rates (Average of Correct Positives and Correct Negatives Rates) with and without the Addition of Eigenregions as Region Features

\begin{tabular}{|c|c|c|}
\hline Class & Rate w/ out eigenregions & Rate w. eigenregions \\
\hline Vegetation & 0.924 & 0.924 \\
\hline Blue Sky & 0.89 & 0.918 \\
\hline Skin & 0.905 & 0.924 \\
\hline
\end{tabular}

TABLE 4

Images Classification Rates (Average of Correct Positives and Correct Negatives Rrates) with Image Semantic Classes

\begin{tabular}{|c|c|c|}
\hline Class & Image Feature Classification & Region Feature Classification \\
\hline Vegetation & 0.81 & 0.89 \\
\hline Blue Sky & 0.8 & 0.91 \\
\hline Skin & 0.70 & 0.85 \\
\hline
\end{tabular}

Region-based classification leads to a better overall image classification.

of the eigenregions is low (see Fig. 5), their potential for discriminating between different classes is still relevant.

\section{From Region to Image Classification}

Let $A_{\nu}$ be a semantic region class ("bluesky," "vegetation," or " skin tones"), $R$ be a region, $S_{v}$ be a semantic image class, and $I$ be the image. If we consider the output of the region classification to be $p\left(A_{\nu} \mid R\right)$, then we have [5], [6]:

$$
p\left(S_{v} \mid I\right)=\sum_{i=1}^{N} \operatorname{area}\left(R_{i}\right) \cdot p\left(A_{\nu} \mid R_{i}\right) \quad \cdot \operatorname{Heaviside}\left(p\left(A_{\nu} \mid R_{i}\right)-\theta\right)
$$

where $\theta$ is the chosen threshold of the multivariate Gaussian.

The performance of this region-based image classification can be assessed by comparing $p\left(S_{v} \mid I\right)$ with the results of a previous classification on the same image set obtained by using image features only [5], [6]. The results are listed in Table 4 .

\section{Conclusion}

We have developed a geometrical feature, using Principal Component Analysis, that combines region area, shape, and position information. Using the eigenregion features in image classification for automatic color correction resulted in an improved classification for images containing localized regions.

Based on our data base of 13,500 real-scene photographs taken by nonprofessionals, a key result obtained from eigenregions is that the largest variance in region geometry is due to the area and not to the shape or position. This implies that a PCA-based method might not be the most adapted for region reconstruction purposes, since the variance is more due to randomness than to a defined structure.

Notwithstanding, using eigenregions in conjunction with color and texture features allows for a noticeable improvement of image classification rates. Despite a low reconstruction rate and random structure, eigenregions can still be used in image classification because they are discriminative enough for a variety of critical image classes.

Eigenregions being database dependent, other results may be obtained. It is possible that for image databases containing more structured scenes, eigenregions may perform even better than in our example.

\section{ACKNOWLEDGMENTS}

The authors would like to thank Dr. Markus Naef and the IIT group of Gretag Imaging AG, Professor Bernt Schiele, ETHZ, and Professor Emre Telatar, EPFL.

\section{REFERENCES}

[1] C. Carson, S. Belongie, H. Greenspan, and J. Malik, "Blobworld: Image Segmentation Using Expectation-Maximization and Its Application to Image Querying," IEEE Trans. on Pattern Analysis and Machine Intelligence, vol. 24, pp. 1026-1038, 2002

[2] S.-F. Chang, "Content-Based Indexing and Retrieval of Visual Information," IEEE Signal Processing Magazine, vol. 14, pp. 45-48, 1997.

[3] Y.Q. Chen, "Novel Techniques for Image Texture Classification," $\mathrm{PhD}$ Thesis, Univ. of Southampton, 1995.

[4] C. Faloutsos et al., "Efficient and Effective Querying by Image Content," J. Intelligent Information Systems, vol. 3, pp. 231-262, 1994.

[5] C. Fredembach, "Salient Regions for Automatic Color Correction and Image Classification," MSc Thesis, EPFL, 2003.

[6] C. Fredembach, M. Schröder, and S. Susstrunk, "Region-Based Image Classification for Automatic Color Correction," Proc. ISET 11th Color Imaging Conf., pp. 59-65, 2003.

[7] C.-S. Fuh, S.-W. Cho, and K. Essig, "Hierarchical Color Image Region Segmentation for Content-Based Image Retreival System," IEEE Trans. Image Processing, vol. 9, pp. 156-162, 2000.

[8] K. Fukunaga, "Statistical Pattern Recognition," Handbook of Pattern Recognition and Computer Vision, C. Chen, L. Pau and P. Wang, eds., pp. 33-60, World Scientific, 1993.

[9] R.M. Haralick, K. Shanmugan, and I. Dinstein, "Textural Features for Image Classification," IEEE Trans. Systems, Man, and Cybernetics, vol. 3, pp. 610621, 1973.

[10] A.K. Jain, Fundamentals of Digital Image Processing. Prentice-Hall Int'1, 1989.

[11] W. Ma and B. Manjunath, "EdgeFlow: A Technique for Boundary Detection and Image Segmentation," IEEE Trans. Image Processing, vol. 9, pp. 13751388, 2000.

[12] MPEG Requirements Group, “Description of MPEG-7 Content Set," ISO/ IEC/JTC1/SC29/WG11/N2467, 2001.

[13] H. Palus, "Region-Based Colour Image Segmentation: Control Parameters and Evaluation Functions," Proc. ISET First European Conf. Color in Graphics, Imaging, and Vision, pp. 259-262, 2002.

[14] T. Pun and D. Squire, "Statistical Structuring of Pictorial Databases for Content-Based Image Retrieval Systems," Pattern Recognition Letters, vol. 17, pp. 1299-1310, 1996.

[15] R. Ramamoorthi, "Analytic PCA Construction for Theoretical Analysis of Lighting Variability in Images of a Lambertian Object," IEEE Trans. Pattern Analysis and Machine Intelligence, vol. 24, pp. 1322-1333, 2002.

[16] M. Schroder and S. Moser, "Automatic Color Correction Based on Generic Content Based Image Analysis," Proc. ISET Ninth Color Imaging Conf., pp. 41-45, 2001.

[17] M. Schröder, "Stochastic Modeling of Image Content in Remote Sensing Image Archives," PhD Thesis, ETHZ, 2000.

[18] A.W.M. Smeulders, M. Worring, S. Santini, A. Gupta, and R. Jain, “ContentBased Image Retrieval at the End of the Early Years," IEEE Trans. Pattern Analysis and Machine Intelligence, vol. 22, pp. 1349-1380, 2000.

[19] M. Turk and A. Pentland, "Eigenfaces for Recognition," J. Cognitive Neuroscience, vol. 3, pp. 71-96, 1991.

[20] P. Smits and A. Annoni, "Toward Specification-Driven Change Detection," IEEE Trans. Geoscience and Remote Sensing, vol. 38, pp. 1484-1488, 2000.

[21] G. Wyszecki and W. Stiles, Color Science: Concept and Methods, Quantitative Data, and Formulae. Wiley, 1982.

$\triangleright$ For more information on this or any other computing topic, please visit our Digital Library at www.computer.org/publications/dlib. 